\title{
Cancer trial assesses clinical benefit of gene-targeted treatment
}

A pilot trial launched on January 30 by the US National Cancer Institute (NCI) aims to evaluate whether genetic screening improves clinical outcomes for patients with advanced solid tumors. The phase 2 Molecular Profiling-based Assignment of Cancer Therapeutics (M-PACT) trial is one of the first such studies to use a randomized design. Cancer centers are beginning to use genetic sequencing of tumors to guide patients' treatment, but there is little unbiased evidence that such an approach works. "We don't know that assigning therapy based on the presence of genetic mutations in tumors does result in clinical benefit for patients with refractory solid tumors," says Shivaani Kummar, head of early clinical trials development at NCI's Division of Cancer Treatment and Diagnosis and M-PACT's principal investigator. "This trial will actually address that question."

For the M-PACT study, 180 patients with advanced tumors resistant to standard therapies will be enrolled after an initial screening. Patients' tumors will be sequenced to look for 391 molecular aberrations in 20 commonly mutated genes known to affect the utility of targeted therapies. All types of solid tumors that can be easily biopsied are considered, but depending on their tumors' genetic profile, patients will be randomly assigned to a treatment arm, in which the drug targets a mutation in their tumors or relevant pathway, or a control arm, where the choice of therapeutic is not guided by genomic information. The researchers will then track how well patients in each group respond to the treatments and how

long that response lasts. Individuals in the nontargeted arm will have the option to cross over to receive therapy targeting their specific

"We are at the point where we have a taxonomy and we have some drugs that can affect the more common mutations." mutation if their disease progresses.

The study will try four different drugs or drug combinations. Two of the four drugs used in the gene-matched treatment groups are approved by the US Food and Drug Administration: Novartis' Afinitor (everolimus), a target of rapamycin inhibitor, and GlaxoSmithKline's Mekinist (trametinib), a

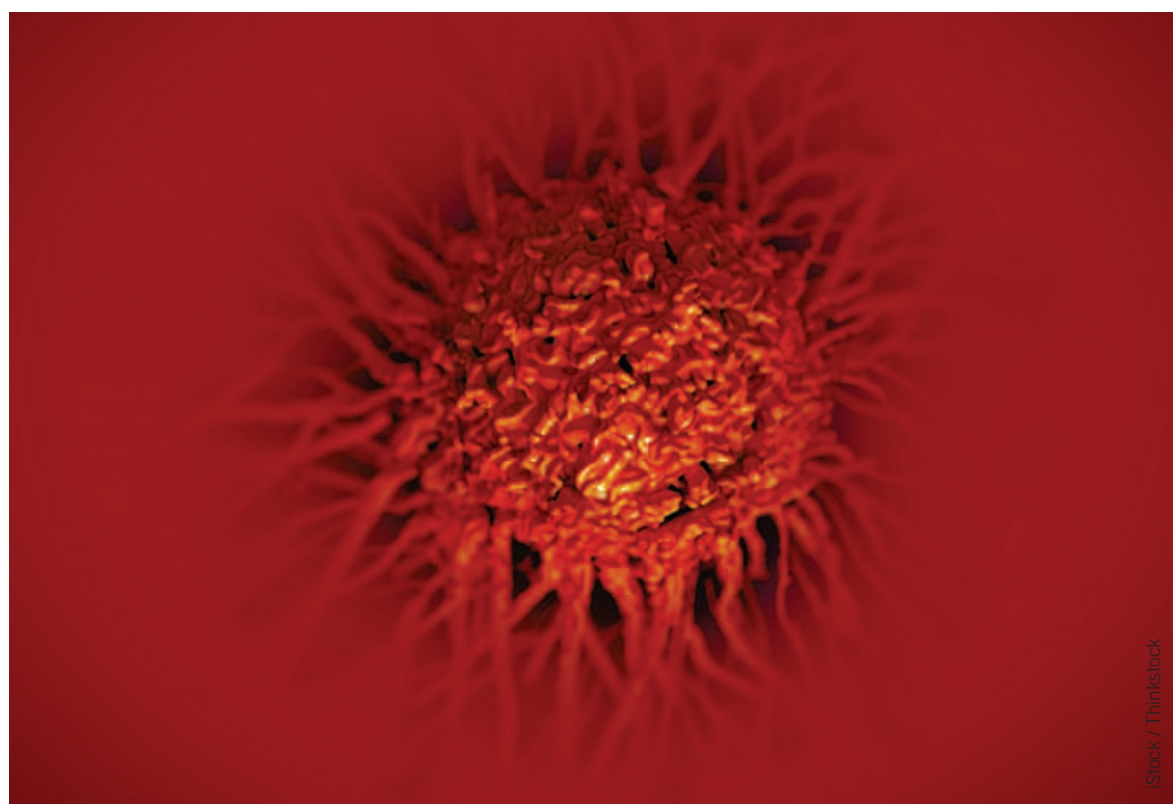

Tumors rarely have one mutation. The benefits of matching treatments with a tumor's genetic profile are now being tested.

mitogen-activated ERK kinase inhibitor. The other two drugs-AbbVie's ABT-888 (veliparib), a poly (ADP-ribose) polymerase inhibitor, and AstraZeneca's MK-1175, a Wee1 tyrosine kinase inhibitor-are not approved, but their dosing, and the fact that they hit their intended target, is known.

Because M-PACT was designed to test multiple drugs in many different mutation tumor types, it should help researchers establish how best to employ such molecular profiling, says Janet Dancey, director of the High Impact Clinical Trials program at the Ontario Institute for Cancer Research, who was not involved in the study. "We are at the point where we have a taxonomy and we have some drugs that can affect the more common mutations, but there are a whole lot of things that we don't know that we are just starting to get to," she says.

For example, patients rarely have just one mutation in their tumor, so which one should a treatment target? How strongly must the drugs interfere with their target pathway to have the necessary effect? Results from trials such as M-PACT, along with efforts such as the International Cancer Genome Consortium, which released data for more than 10,000 cancer genomes in February to help catalog cancer mutations and track their outcomes in patients, will start to bring such issues into focus, experts hope.

One key question is how researchers will interpret the trial if it does indeed show that patients do better when treatments are matched to mutations. Such a result would arguably confirm the broader strategy of genomically targeting cancer therapy, says Razelle Kurzrock, vice-chief of hematologyoncology at the University of California San Diego Moores Cancer Center. Dancey, however, advocates a more limited approach, in which different drugs and targets will have to be tested separately. Meanwhile, if the study fails, researchers will have to carefully assess whether the mutations or patient population were chosen incorrectly. "Even if it's negative, this study will offer a wealth of information that will guide the next phase of trials," says Kummar.

Alla Katsnelson Northampton, Massachusetts 